\title{
La Evaluación del Desempeño por Competencias: Percepciones de Docentes y Estudiantes en la Educación Superior
}

\author{
Domingos J. Fernandes ${ }^{(1) *}$, Maria Sotolongo ${ }^{(2)}$ y Carlos C. Martínez ${ }^{(3)}$ \\ (1) Universidad José Eduardo dos Santos, Rua Sousa Neves, Cidade Baixa, Edifício Enicar, 3ํ. Piso, \\ Huambo - Angola. (e-mail: jfernandes07@yahoo.com.br) \\ (2) Facultad de Ingeniería Mecánica e Industrial. Universidad Central "Marta Abreu" de Las Villas, \\ Villa Clara - Cuba. (e-mail: msotolongo@uclv.edu.cu) \\ (3) Facultad de Ciencias Económicas y Turismo. Universidad Central "Marta Abreu" de Las Villas, \\ Villa Clara - Cuba. (e-mail: cristobalc@uclv.edu.cu)
}

${ }^{*}$ Autor a quien debe ser dirigida la correspondencia

Recibido Feb. 23, 2016; Aceptado Abr. 11, 2016; Versión final May. 19, 2016, Publicado Oct. 2016

\begin{abstract}
Resumen
El objetivo de la investigación fue conocer las percepciones de estudiantes y docentes sobre el instrumento para evaluar el desempeño docente en universidades públicas angolanas y el orden de prioridad que le confieren a cada dimensión. El estudio se realizó en la Universidad José Eduardo dos Santos, institución representativa del universo a investigar. El instrumento de evaluación está estructurado en 23 afirmaciones distribuidas en cuatro dimensiones: competencias pedagógicas-didácticas, investigativas, de gestión académica y de extensión universitaria. Este instrumento se aplicó a una muestra conformada por 126 docentes y 342 estudiantes. El análisis de las percepciones de estudiantes y profesores permitió concluir sobre la efectividad de utilizar las cuatro dimensiones propuestas para evaluar el desempeño docente. De acuerdo con las opiniones de ambos grupos la dimensión de las competencias pedagógicas-didácticas ocupa la primera posición.
\end{abstract}

Palabras clave: evaluación del desempeño; competencias; percepciones; estudiantes; profesores

\section{The Performance Evaluation by Competences: Perceptions of Teachers and Students in Higher Education}

\begin{abstract}
The objective of the research was to know the perceptions of students and teachers on the instrument employed to evaluate teaching performance in Angolan public universities and the order of priority that they confer to each dimension. The study was conducted in the University José Eduardo dos Santos, an institution considered to represent public Angolan universities. The evaluation instrument was structured into 23 statements distributed in four dimensions: pedagogical and didactic competences, research activities, academic management skills and university extension. This instrument was applied to a sample composed of 126 teachers and 342 students. The analysis of the perceptions of students and teachers concluded on the effectiveness of using the proposed four dimensions to evaluate teacher performance. According to the opinions of both groups the dimension called pedagogical-didactic competencies occupies the first position.
\end{abstract}


$\underline{\text { La Evaluación del Desempeño por Competencias: Percepciones de Docentes y Estudiantes } \quad \text { Fernandes }}$

\section{INTRODUCCIÓN}

Dados los cambios y transformaciones que ha sufrido la educación superior en la actualidad a nivel mundial, Ospina (2006) plantea que las universidades se enfrentan a desafíos en formar en los estudiantes las competencias necesarias que les permitan desempeñarse en los diversos campos laborales. Este paradigma impone condiciones de exigencias respecto a la competitividad y vigencia de los conocimientos de los profesionales graduados. Por tanto, el docente universitario es uno de los responsables de estimular en los estudiantes los conocimientos propios de la disciplina que enseña y las competencias que son necesarias para la adaptación en el mundo laboral (Daura, 2011). En este sentido, se ha podido constatar en importantes contribuciones de prestigiosos autores e investigadores, la importancia de la evaluación del desempeño del docente universitario para la mejora de la calidad del proceso de enseñanza aprendizaje. Dada la diversidad de funciones del profesor universitario su evaluación deberá incluir no solo la docencia, evitando privilegiarla en tanto que este personal desempeña otras funciones como la gestión social, la administración y la investigación (Cancino et al., 2015; Coelho y Vásquez, 2008; Creus et al., 2011; De León y Huerta, 2010; Páramo, 2008; Tirado et al., 2007 y Torra et al., 2012).

La evaluación del desempeño docente en la educación superior se ha realizado por diversos procedimientos y métodos, tales como: la autoevaluación del profesor, la evaluación por pares y responsables académicos y la evaluación por los estudiantes, por solo citar algunos de los procedimientos más difundidos (Tobón, 2010; Moreno et al., 2014). Si bien con el paso del tiempo se ha buscado tener más referentes de cómo evaluar el desempeño docente, sigue dominando el uso de cuestionario de opinión de estudiantes (hoy en día incluso en línea), lo anterior, se justifica tomando en consideración las dimensiones poblacionales estudiantiles y docentes que son parte del proceso (Moreno et al., 2014). Según Marsh (2007) los estudiantes son fuente evaluadora confiable y válida de la eficacia del desempeño docente, por lo que la recomienda para los propósitos de mejorar la enseñanza, la toma de decisiones sobre el personal académico y salvaguardar el control de la calidad educativa. Si verdaderamente hablamos de una evaluación integral de la compleja actividad docente del profesor consideramos no se le debe restar importancia a la opinión de los que directamente reciben el producto del desempeño docente y que son la razón de ser de su existencia (Pimienta, 2014). Por otra parte, Fernández (2011) considera que los profesores no mejorarán su enseñanza, asumiendo una perspectiva profesional de su tarea, si no someten su enseñanza y sus prácticas educativas a procesos de evaluación que orienten la mejora y que, incluso, reconozcan la calidad docente.

En la literatura científica consultada se constatan varios estudios realizados para medir la efectividad del docente desde la perspectiva de los estudiantes (Coelho y Vásquez, 2008; Moreno et al., 2014; Páramo 2008; Pimienta, 2014; Rocha, 2013 y Tirado et al., 2007) y el orden de prioridad que otorgan a cada dimensión definida para la evaluación del docente (Cortés et al., 2014) mientras en menor medida se aprecian estudios que comparen las percepciones de estudiantes y profesores sobre el desempeño docente. Sin embargo, Tobón (2010) destaca que desde el enfoque socio formativo de las competencias, el énfasis no está en los estudiantes, ni tampoco en los docentes, sino en la relación intersistémica de ambos. Prolongando esta línea de pensamiento, el presente artículo centra su atención en conocer la opinión de estudiantes y docentes sobre el instrumento para evaluar el desempeño docente en universidades públicas angolanas, sin menospreciar los criterios de los pares y los responsables académicos.

Angola no está ajena a esta situación, actualmente la evaluación del desempeño de los recursos humanos y especialmente de los docentes en las universidades públicas angolanas ha sido una preocupación permanente, motivada por la necesidad de elevar los niveles de calidad de las clases y en general del trabajo de los docentes universitarios y consecuentemente contribuir a mejorar el proceso de enseñanzaaprendizaje. Estudios previos realizados por Nascimento (2005) y Chimpolo (2015) sobre la situación del sistema de enseñanza superior en Angola revelaron todo un conjunto de debilidades que obstaculizan el buen desempeño de los docentes universitarios, entre ellas se destacan: los docentes encargados de formar el capital humano prácticamente no poseen desde el punto de vista pedagógico una preparación adecuada, adolecen de una cultura general, así como de conocimiento sobre didáctica y técnicas de enseñanza y evaluación; además se muestran poco comprometidos en el desempeño de su profesión porque los administrativos evalúan a todos con un sistema de evaluación del desempeño (E.D) que no permite establecer distinciones entre los docentes de un desempeño superior con relación a los de un desempeño insatisfactorio; la gran mayoría de los docentes aspiran a un mayor nivel académico, pues esto le facilita tener más ingresos financieros y no están preocupados o interesados en mejorar sustancialmente la calidad del proceso de enseñanza-aprendizaje y carecen de habilidades pedagógicas y didácticas, en ocasiones no actúan como facilitadores del aprendizaje y por tanto no contribuyen a desarrollar en los educandos la creatividad. Sin embargo, estas competencias tampoco se les evalúan en su desempeño. 
Además, investigaciones realizadas por Fernandes, Martínez y Sotolongo (2015) pusieron en evidencia insuficiencias en la E.D en la Universidad José Eduardo dos Santos (UJES), institución representativa de las universidades públicas angolanas; tales como: el instrumento para la evaluación del desempeño de los docentes universitarios es el mismo que se utiliza para evaluar a los demás funcionarios públicos (personal técnico-administrativo), esto trae consigo que los indicadores definidos en este instrumento sean muy generales y no permitan evaluar las competencias que se deben medir en el desempeño de un docente universitario, obteniéndose resultados muy subjetivos que provocan insatisfacciones y desempeños que no potencian la mejora de las competencias del personal docente, situación que incide negativamente en la calidad del proceso de enseñanza-aprendizaje en las universidades públicas angolanas. Los indicadores definidos para la evaluación del desempeño son: competencia profesional, el dinamismo e iniciativa, el cumplimiento de tareas, las relaciones humanas en el trabajo, la adaptación profesional, el asiduidad, la puntualidad, la disciplina, la utilización adecuada de los medios de trabajo, la presentación y postura individual.

Esta problemática, justificó, la necesidad de desarrollar un sistema de evaluación del desempeño que permitiera un cambio en el quehacer cotidiano de los docentes universitarios angolanos. Sin embargo, muchas de las universidades públicas angolanas no sabían realmente cómo evaluar el desempeño de sus docentes, pues no abundaban instrumentos metodológicos pertinentes que permitieran evaluarlos y mucho menos proyectar acciones de mejora de sus competencias para contribuir a la calidad del proceso de enseñanza-aprendizaje en estas instituciones educativas.

A pesar de la existencia de la literatura foránea sobre el tema de la evaluación del desempeño, particularmente los aportes de Portugal et al. (2014), Gomes et al. (2014), Martínez (2013) y Shiroma et al. (2013), no aparece en esta un análisis metodológico del tema que permita a las universidades públicas angolanas, evaluar el desempeño de sus docentes en las condiciones específicas de su desarrollo. La mayoría de esta literatura pretende generalizar los sistemas de evaluación del desempeño desarrollados por algunas instituciones en determinado contexto (Cruz et al., 2014; Galvão et al., 2011; Murrillo et al., 2012 y Sandoval, 2014;); por lo que aplicar estos sistemas en las condiciones de las universidades públicas angolanas pudiera resultar un fracaso. La problemática expuesta, justifica en gran medida, la necesidad de conocer las percepciones de profesores y estudiantes sobre la evaluación del desempeño docente por competencias utilizando el instrumento por dimensiones que se aplica en la UJES.

\section{MATERIALES Y MÉTODOS}

\section{Población y muestra}

De una población conformada por 187 docentes, y 3089 estudiantes se determinó el tamaño de la muestra, utilizando los criterios de Cochram (1999) y De Veaux (2001) para garantizar la representatividad de la población en la muestra tomada, esto permite que exista fidelidad y exactitud en la información recopilada. Obteniéndose como resultado una muestra conformada por 126 docentes y 342 estudiantes universitarios (ver tabla 1).

Tabla1: Descripción de los componentes de la ecuación para calcular el tamaño de la muestra.

\begin{tabular}{|c|c|c|c|}
\hline & & Docentes & Estudiantes \\
\hline$n$ & Tamaño de la muestra & 126 & 342 \\
\hline $\mathrm{N}$ & Tamaño de la población & 187 & 3089 \\
\hline $\mathrm{Z} 1-\propto / 2$ & Percentil de la distribución normal & 1.96 & 1.96 \\
\hline$p$ & Valor que da el mayor tamaño de la muestra & 0.50 & 0.50 \\
\hline$d$ & Error absoluto & 0.05 & 0.05 \\
\hline
\end{tabular}

\section{Procedimiento}

El instrumento de evaluación (Tabla 2), permitió a la UJES, realizar la evaluación del desempeño por competencias de sus docentes. El instrumento se elaboró de acuerdo con los ámbitos de trabajo y desempeño académico de los docentes en las universidades públicas angolanas y las experiencias de estudios precedentes realizados por Cardoso et al. (2013), Martínez at al. (2012), Mas (2014), Matos et al. (2012), Moran et al. (2015), Nava et al. (2013), Pimienta (2014), Torra et al. (2012) y Tobón (2013). En este sentido, teniendo en cuenta que el concepto de competencia ha sido definido por diversos autores (Tobón, 2006 y Zabala y Arnau, 2007), los cuales coinciden en que es un término holístico que incluye habilidades, destrezas y actitudes en un contexto especifico en donde se utilizan no solo en forma conceptual y procedimental si no también actitudinal, pero considerando que estos autores abordan diferentes enfoques de competencias: conceptual, técnico y cultural. En este estudio se utiliza el enfoque expresado por Zabalza 
(2012) centrado en el aspecto técnico porque involucra la forma de implementar la propuesta como modo de actuación docente factible de ser evaluada.

Tabla 2: Instrumento de evaluación del desempeño docente en la UJES. La escala de evaluación para todas las dimensiones y criterios de desempeño es: 5 . Muy Alto, 4. Alto, 3. Medio, 2. Bajo, 1.Muy Bajo

\begin{tabular}{|c|c|}
\hline Dimensiones & Criterios de desempeño \\
\hline \multirow{10}{*}{$\begin{array}{l}\text { Competencias } \\
\text { pedagógicas- didácticas }\end{array}$} & Dominar el contenido de la asignatura y las competencias a desarrollar. \\
\hline & Estructurar lógicamente la presentación de sus ideas de forma oral y escrita. \\
\hline & Favorecer el enfoque pedagógico en la asignatura. \\
\hline & Participar en el diseño curricular propiciando la interdisciplinariedad. \\
\hline & Elaborar materiales de apoyo didáctico en soporte convencional o TIC. \\
\hline & $\begin{array}{l}\text { Diseñar y desarrollarlas actividades académicas de forma que propicien el } \\
\text { autoaprendizaje y el desarrollo de competencias profesionales y personales. }\end{array}$ \\
\hline & $\begin{array}{l}\text { Emplear la evaluación diagnóstica, sumativa y formativa proporcionando } \\
\text { retroalimentación de la evaluación a los estudiantes. }\end{array}$ \\
\hline & $\begin{array}{l}\text { Realizar la planeación didáctica de las prácticas profesionales y participar en su } \\
\text { supervisión. }\end{array}$ \\
\hline & $\begin{array}{l}\text { Aplicar estrategias metodológicas de aprendizaje y evaluación adecuada a las } \\
\text { necesidades de los estudiantes teniendo en cuenta el uso de las TIC. }\end{array}$ \\
\hline & $\begin{array}{l}\text { Ser tutor del proceso de aprendizaje del estudiante y establecer las relaciones y la } \\
\text { comunicación interpersonal que reclama esta función. }\end{array}$ \\
\hline \multirow[t]{5}{*}{$\begin{array}{l}\text { Competencias } \\
\text { investigativas }\end{array}$} & $\begin{array}{l}\text { Desarrollar proyectos de investigación que permitan realizar trabajos colaborativos } \\
\text { con sus estudiantes. }\end{array}$ \\
\hline & $\begin{array}{l}\text { Publicar artículos científicos mediante trabajo colaborativo con sus estudiantes para } \\
\text { mantener los estándares profesionales y estar al corriente de los avances del } \\
\text { conocimiento. }\end{array}$ \\
\hline & Participar en congresos y eventos propiciando la participación de sus estudiantes. \\
\hline & $\begin{array}{l}\text { Cursar becas predoctorales o postdoctorales para potenciar el compromiso científico } \\
\text { con la disciplina. }\end{array}$ \\
\hline & $\begin{array}{l}\text { Realizar pasantías en instituciones educativas que permitan realizar trabajos } \\
\text { colaborativos con sus estudiantes. }\end{array}$ \\
\hline \multirow[t]{3}{*}{$\begin{array}{l}\text { Competencias de gestión } \\
\text { Académica }\end{array}$} & $\begin{array}{l}\text { Coordinar y/o participar en equipos interdisciplinarios para gestionar proyectos } \\
\text { docentes. }\end{array}$ \\
\hline & $\begin{array}{l}\text { Participar en consejos académicos y comisiones colaborando con la gestión de la } \\
\text { institución. }\end{array}$ \\
\hline & $\begin{array}{l}\text { Participar en reuniones institucionales reglamentadas manteniendo relaciones de } \\
\text { respeto y colaboración. }\end{array}$ \\
\hline \multirow{5}{*}{$\begin{array}{l}\text { Competencias de } \\
\text { Extensión universitaria }\end{array}$} & Promover actividades con la comunidad. \\
\hline & Dirigir y/o participar en proyectos de extensión social y cultural de la institución. \\
\hline & $\begin{array}{l}\text { Dirigir y/o participar en proyectos de capacitación, consultorías y pasantías de } \\
\text { profesores y estudiantes. }\end{array}$ \\
\hline & Dirigir y/o participar en proyectos de cooperación con otras áreas de la universidad. \\
\hline & $\begin{array}{l}\text { Participar en actividades con organizaciones vinculadas a la educación, la ciencia, la } \\
\text { tecnología y la cultura. }\end{array}$ \\
\hline
\end{tabular}

Como se puede apreciar en la tabla 2, el instrumento elaborado se estructuró en 23 criterios de desempeño, distribuidos en cuatro dimensiones: competencias pedagógicas-didácticas, investigativas, de gestión académica y de extensión universitaria. La primera dimensión se refirió a las competencias que están relacionadas con el desempeño académico (sus estrategias didácticas, instrumentos de evaluación y su papel como tutores durante los procesos formativos de los estudiantes). La segunda está referida a la investigación como eje transversal en el proceso docente educativo (asesoría de tesis, trabajo colaborativo entre el docente con sus estudiantes y la participación en la escritura de textos académicos). La tercera 
dimensión se refirió a la coordinación y/o participación en equipos interdisciplinarios para gestionar proyectos docentes y asumir un compromiso institucional y social, donde el equipo docente en colaboración con los estudiantes se asuma como la vía para dar respuesta a las múltiples demandas que el contexto genera. Mientras que la cuarta dimensión se refirió al ámbito de la vinculación con la colectividad cómo es su participación en programas de extensión cultural o técnica, la cooperación con programas de desarrollo de la comunidad y con otras áreas de la universidad. Estas dimensiones se miden en una escala Likert, con cinco opciones de evaluación: 1. Muy Bajo (no aporta a la medición del desempeño de los docentes); 2 . Bajo (quizás podría servir para medir el desempeño de los docentes); 3. Medio (útil para medir el desempeño de los docentes); 4. Alto (muy útil para medir el desempeño de los docentes) y 5. Muy Alto (imprescindible para medir el desempeño de los docentes).Además, su fiabilidad se constató mediante el estadístico Alfa de Cronbach y la correspondiente prueba $Q$ de Cochran (Tablas 3 y 4). El Alfa de Cronbach del instrumento fue de 0.842 lo cual significa que es confiable.

Tabla 3: Prueba de fiabilidad Alfa de Cronbach

\begin{tabular}{|c|c|}
\hline \multicolumn{2}{|c|}{ Estadísticos de fiabilidad } \\
\hline Alfa de Cronbach & $\mathrm{N}$ de elementos \\
\hline 0.842 & 4 \\
\hline
\end{tabular}

Tabla 4: Prueba Q de Cochran

\begin{tabular}{|l|l|c|c|c|c|c|}
\hline \multicolumn{7}{|c|}{ ANOVA con la prueba Q de Cochran } \\
\hline \multicolumn{2}{|l|}{} & Suma de cuadrados & gl & Media cuadrática & Q de Cochran & Sig. \\
\hline \multirow{2}{*}{ Inter-personas } & 1346.942 & 467 & 2.884 & & \\
\hline \multirow{3}{*}{$\begin{array}{l}\text { Intra- } \\
\text { personas }\end{array}$} & Inter-elementos & 14.788 & 3 & 4.929 & 31.772 & 0.000 \\
\cline { 2 - 7 } & Residual & 638.712 & 1401 & 0.456 & & \\
\cline { 2 - 7 } & Total & 653.500 & 1404 & 0.465 & & \\
\hline Total & 2000.442 & 1871 & 1.069 & & \\
\hline \multicolumn{2}{|l|}{ Media global $=3.61$}
\end{tabular}

\section{RESULTADOS Y DISCUSIÓN}

Los resultados se presentan para cada una de las dimensiones por separado y se analizan desde dos puntos de vista: las percepciones de los docentes y de los estudiantes y el orden de prioridad que cada grupo otorga a cada dimensión. Para un análisis más exhaustivo del comportamiento de las valoraciones de los docentes respecto a las valoraciones de los estudiantes, se realizaron las tablas de contingencias para cada una de las dimensiones respectivamente, donde se reflejaron las frecuencias correspondientes a cada valor de la escala, así como los porcientos que las mismas representan respecto al total de cada fila(profesores y estudiantes).Para corroborar si estas diferencias son o no significativas se realizó la prueba Chi-cuadrado, mediante la cual se rechaza la hipótesis nula de que "no existen diferencias significativas" si la significación asintótica es menor que 0,05. Asimismo, este nivel de significación se complementó con el análisis delas medidas simétricas, en particular $V$ de Cramer. El procesamiento estadístico se realizó utilizando la paquetería Statistical Packagefor de Social Sciencs (SPSS V20).Los resultados de la percepción de las competencias pedagógicas-didácticas, la prueba Chi-cuadrado y $\mathrm{V}$ de Cramer se muestran en las tablas 5,6 y 7 respectivamente.

Tabla 5: Percepción del impacto de las competencias pedagógicas - didácticas en la evaluación docente.

\begin{tabular}{|l|l|c|c|c|c|c|c|}
\hline \multirow{2}{*}{ Estratos } & Frecuencias y \% & \multicolumn{6}{|c|}{ Escalas } \\
\cline { 3 - 8 } & & Muy Bajo & Bajo & Medio & Alto & Muy Alto & Total \\
\hline \multirow{2}{*}{ Estudiantes } & Frecuencias & 16 & 18 & 96 & 143 & 69 & 342 \\
\cline { 2 - 8 } & \% de estudiantes & 4.7 & 5.3 & 28.1 & 41.8 & 20.2 & 100.0 \\
\hline \multirow{2}{*}{ Docentes } & Frecuencias & 0 & 1 & 35 & 60 & 30 & 126 \\
\cline { 2 - 8 } & $\%$ de docentes & 0.0 & 0.8 & 27.8 & 47.6 & 23.8 & 100.0 \\
\hline \multirow{2}{*}{ Total } & Frecuencias & 16 & 19 & 131 & 203 & 99 & 468 \\
\cline { 2 - 8 } & \% total & 3.4 & 4.1 & 28.0 & 43.4 & 21.2 & 100.0 \\
\hline
\end{tabular}

Tabla 6: Resultados de la prueba Chi-cuadrado para las competencias pedagógicas - didácticas

\begin{tabular}{|l|c|c|c|}
\hline & Valor & gl & Sig. asintótica (bilateral) \\
\hline Chi-cuadrado de Pearson & 11.719 & 4 & .020 \\
\hline Razón de verosimilitudes & 17.383 & 4 & .002 \\
\hline Asociación lineal por lineal & 7.404 & 1 & .007 \\
\hline N de casos válidos & 468 & & \\
\hline
\end{tabular}


Tabla 7: Resultados de las medidas simétricas para las competencias pedagógicas - didácticas.

\begin{tabular}{|l|l|l|c|}
\hline & & Valor & Sig. aproximada \\
\hline \multirow{2}{*}{ Nominal por nominal } & Phi & .158 & .020 \\
\cline { 2 - 4 } & V de Cramer & .158 & .020 \\
\hline N de casos válidos & & 468 \\
\hline
\end{tabular}

Como se puede apreciar, según las percepciones del $62 \%$ de los estudiantes y el $71 \%$ de los docentes consideran que las competencias pedagógicas-didácticas tienen un impacto entre alto y muy alto en la evaluación del desempeño de los docentes en la UJES, aunque se observa un comportamiento similar entre las respuestas de estudiantes y profesores respecto a la percepción del impacto de estas competencias en la evaluación se puede afirmar que existen diferencias significativas entre estudiantes y docentes, dado que el valor de significación asintótica bilateral es menor que 0,05 (véase tabla 6). Mientras $\mathrm{V}$ de Cramer por su parte indica que este nivel de relación es relativamente bajo $(0,158)$ valor que es menor que 0,5 lo que justifica las ligeras diferencias en los comportamientos entre ambos grupos.

La percepción de acuerdo con el criterio de estudiantes y docentes de la segunda dimensión referida a las ccompetencias investigativas se observan en las tablas 8,9 y 10 . Evidenciándose similitud de comportamientos entre la percepción de ambos grupos con relación a las competencias investigativas, el $54,9 \%$ de los estudiantes y el $53,9 \%$ de los docentes así lo corroboran, no existiendo diferencias significativas entre las valoraciones dadas por estudiantes y docentes, dado que la significación asintótica bilateral es mayor que 0,05 como se aprecia en la tabla 9. La percepción de estudiantes y docentes de las competencias de gestión académica (tercera dimensión) se muestran en las tablas 11, 12 y 13 respectivamente.

Tabla 8: Percepción del impacto de las competencias investigativas en la evaluación docente

\begin{tabular}{|c|c|c|c|c|c|c|c|}
\hline \multirow[t]{2}{*}{ Estratos } & \multirow[t]{2}{*}{ Frecuencias y \% } & \multicolumn{6}{|c|}{ Escalas } \\
\hline & & Muy Bajo & Bajo & Medio & Alto & Muy Alto & Total \\
\hline \multirow{2}{*}{ Estudiantes } & Frecuencias & 22 & 47 & 86 & 126 & 61 & 342 \\
\hline & $\%$ de estudiantes & 6.5 & 13.8 & 25.1 & 37.0 & 17.9 & 100.0 \\
\hline \multirow{2}{*}{ Docentes } & Frecuencias & 3 & 13 & 42 & 40 & 28 & 126 \\
\hline & $\%$ de docentes & 2.4 & 10.3 & 33.3 & 31.7 & 22.2 & 100.0 \\
\hline \multirow{2}{*}{ Total } & Frecuencias & 25 & 60 & 128 & 166 & 89 & 468 \\
\hline & $\%$ total & 5.4 & 12.8 & 27.4 & 35.5 & 19.1 & 100.0 \\
\hline
\end{tabular}

Tabla 9: Resultados de la prueba Chi-cuadrado para las competencias investigativas

\begin{tabular}{|l|c|c|c|}
\hline & Valor & Gl & $\begin{array}{c}\text { Sig. asintótica } \\
\text { (bilateral) }\end{array}$ \\
\hline Chi-cuadrado de Pearson & 7.535 & 4 & .110 \\
\hline Razón de verosimilitudes & 7.966 & 4 & .093 \\
\hline Asociación lineal por lineal & 1.759 & 1 & .185 \\
\hline N de casos válidos & 468 & & \\
\hline
\end{tabular}

Tabla 10: Resultados de las medidas simétricas para las competencias investigativas

\begin{tabular}{|l|l|c|c|}
\hline \multirow{2}{*}{ Nominal por nominal } & & Valor & Sig. aproximada \\
\cline { 2 - 4 } & Phi & .127 & .110 \\
\cline { 2 - 4 } & V de Cramer & .127 & .110 \\
\hline N de casos válidos & & 468 \\
\hline
\end{tabular}

Tabla 11: Percepción del impacto de las competencias de gestión académica en la evaluación docente

\begin{tabular}{|c|c|c|c|c|c|c|c|}
\hline \multirow[t]{2}{*}{ Estratos } & \multirow[t]{2}{*}{ Frecuencias y \% } & \multicolumn{6}{|c|}{ Escalas } \\
\hline & & Muy Bajo & Bajo & Medio & Alto & Muy Alto & Total \\
\hline \multirow{2}{*}{ Estudiantes } & Frecuencias & 27 & 23 & 88 & 125 & 79 & 342 \\
\hline & $\%$ de estudiantes & 7.9 & 6.7 & 25.7 & 36.5 & 23.1 & 100.0 \\
\hline \multirow{2}{*}{ Docentes } & Frecuencias & 1 & 7 & 45 & 56 & 17 & 126 \\
\hline & $\%$ de docentes & 0.8 & 5.6 & 35.7 & 44.4 & 13.5 & 100.0 \\
\hline \multirow{2}{*}{ Total } & Frecuencias & 28 & 30 & 133 & 181 & 96 & 468 \\
\hline & $\%$ total & 6.0 & 6.4 & 28.4 & 38.7 & 20.5 & 100.0 \\
\hline
\end{tabular}

Tabla 12: Resultados de la prueba Chi-cuadrado para las competencias de gestión académica

Tabla 12: Resultados de la prueba Chi-cuadrado para las competencias de gestión académica
\begin{tabular}{|l|c|c|c|}
\hline & Valor & Gl & $\begin{array}{c}\text { Sig. asintótica } \\
\text { (bilateral) }\end{array}$ \\
\hline Chi-cuadrado de Pearson & 16.813 & 4 & .002 \\
\hline Razón de verosimilitudes & 20.175 & 4 & .000 \\
\hline Asociación lineal por lineal & 0.133 & 1 & .715 \\
\hline N de casos válidos & 468 & & \\
\hline
\end{tabular}


Tabla 13: Resultados de las medidas simétricas para las competencias de gestión académica

\begin{tabular}{|l|l|c|c|}
\hline & & Valor & Sig. aproximada \\
\hline \multirow{2}{*}{ Nominal por nominal } & Phi & .190 & .002 \\
\cline { 2 - 4 } & V de Cramer & .190 & .002 \\
\hline N de casos válidos & & 468 \\
\hline
\end{tabular}

La percepción de estudiantes y docentes con relación a las competencias de gestión académica presentó un comportamiento similar para ambos grupos, el 59,6\% de los estudiantes y el 57,9\% de los docentes así lo corroboran, no existiendo diferencias significativas entre las valoraciones dadas por estudiantes y docentes, dado que la significación asintótica bilateral es mayor que 0,05 (véase tabla 12). El análisis de las percepciones sobre la cuarta dimensión referida a las competencias de extensión universitaria mostró un comportamiento similar a las competencias investigativas y de gestión académica (véase tablas 14, 15 y 16).

Tabla 14: Percepción del impacto de las competencias de extensión universitaria en la evaluación docente

\begin{tabular}{|l|l|c|c|c|c|c|c|}
\hline \multirow{2}{*}{ Estratos } & Frecuencias y \% & \multicolumn{7}{|c|}{ Escalas } \\
\cline { 3 - 8 } & & Muy Bajo & Bajo & Medio & Alto & Muy Alto & Total \\
\hline \multirow{2}{*}{ Estudiantes } & Frecuencias & 17 & 26 & 101 & 136 & 62 & 342 \\
\cline { 2 - 8 } & $\%$ de estudiantes & 5.0 & 7.6 & 29.5 & 39.8 & 18.1 & 100.0 \\
\hline \multirow{2}{*}{ Docentes } & Frecuencias & 3 & 9 & 45 & 48 & 21 & 126 \\
\cline { 2 - 8 } & $\%$ de docentes & 2.4 & 7.1 & 35.7 & 38.1 & 16.7 & 100.0 \\
\hline \multirow{2}{*}{ Total } & Frecuencias & 20 & 35 & 146 & 184 & 83 & 468 \\
\cline { 2 - 8 } & $\%$ total & 4.3 & 7.5 & 31.2 & 39.3 & 17.7 & 100.0 \\
\hline
\end{tabular}

Tabla 15: Resultados de la prueba Chi-cuadrado para las competencias de extensión universitaria

\begin{tabular}{|l|c|c|c|}
\hline & Valor & Gl & $\begin{array}{c}\text { Sig. asintótica } \\
\text { (bilateral) }\end{array}$ \\
\hline Chi-cuadrado de Pearson & 2.775 & 4 & .596 \\
\hline Razón de verosimilitudes & 2.930 & 4 & .570 \\
\hline Asociación lineal por lineal & .010 & 1 & .920 \\
\hline N de casos válidos & 468 & & \\
\hline
\end{tabular}

Tabla 16: Resultados de las medidas simétricas para las competencias de extensión universitaria

\begin{tabular}{|l|l|c|c|}
\hline & & Valor & Sig. aproximada \\
\hline Nominal por nominal & Phi & .077 & .596 \\
\cline { 2 - 4 } & V de Cramer & .077 & .596 \\
\hline N de casos válidos & & 468 \\
\hline
\end{tabular}

Para determinar globalmente la valoración de las percepciones de estudiantes y docentes a las distintas competencias se determinó un índice ponderado a partir de multiplicar por 1, 2, 3, 4 y 5 las frecuencias correspondientes a "Muy bajo", "Bajo", "Medio", "Alto" y "Muy Alto". Posteriormente la suma de los valores alcanzado para cada competencia se dividió por el máximo valor posible a alcanzar, en el caso de los estudiantes (1710) y en el de los docentes (630), el índice resultante es un valor comprendido entre 0 y 1 , con los que se puede realizar cualquier comparación y valoración. Los resultados obtenidos se muestran en las tablas 17 y 18.

Tabla 17: Resultado de las percepciones de los estudiantes

\begin{tabular}{|l|c|c|c|c|c|c|}
\hline \multirow{2}{*}{ Dimensiones } & \multicolumn{5}{c|}{ Frecuencias } \\
\cline { 2 - 7 } & Muy Bajo & Bajo & Medio & Alto & Muy Alto \\
\hline Competencias pedagógico-didácticas & 16 & 18 & 96 & 143 & 69 \\
\hline Competencias investigativas & 22 & 47 & 86 & 126 & 61 \\
\hline Competencias de la gestión académica & 27 & 23 & 88 & 125 & 79 \\
\hline Competencias de extensión universitaria & 17 & 26 & 101 & 136 & \multicolumn{2}{c|}{62} \\
\hline \\
\hline
\end{tabular}

Según las percepciones de estudiantes y docentes, la competencia que consideran más importante en la evaluación del desempeño docente es la competencia pedagógico-didáctica, es decir que el profesor facilite el aprendizaje. El orden de prioridad otorgado según las percepciones de los estudiantes concuerda con los resultados obtenidos por Coelho y Vásquez (2008) y Cortés et al. (2014). El segundo lugar en importancia presenta diferencias de acuerdo a las percepciones de estudiantes y docentes. 
Tabla 18: Resultado de las percepciones de los docentes

\begin{tabular}{|c|c|c|c|c|c|c|}
\hline \multirow{2}{*}{ Dimensiones } & \multicolumn{6}{|c|}{ Frecuencias } \\
\hline & Muy Bajo & Bajo & Medio & Alto & \multicolumn{2}{|c|}{ Muy Alto } \\
\hline Competencias pedagógico-didácticas & 0 & 1 & 35 & 60 & \multicolumn{2}{|c|}{30} \\
\hline Competencias investigativas & 3 & 13 & 42 & 40 & \multicolumn{2}{|c|}{28} \\
\hline Competencias de la gestión académica & 1 & 7 & 45 & 56 & \multicolumn{2}{|c|}{17} \\
\hline Competencias de extensión universitaria & 3 & 9 & 45 & 48 & \multicolumn{2}{|c|}{21} \\
\hline & \multicolumn{5}{|c|}{ Ponderación de las frecuencias } & Índice \\
\hline Competencias pedagógico-didácticas & 0 & 2 & 105 & 240 & 150 & 0.79 \\
\hline Competencias investigativas & 3 & 26 & 126 & 160 & 140 & 0.72 \\
\hline Competencias de la gestión académica & 1 & 14 & 135 & 224 & 85 & 0.73 \\
\hline Competencias de extensión universitaria & 3 & 18 & 135 & 192 & 105 & 0.72 \\
\hline
\end{tabular}

Los estudiantes consideran que deben priorizarse las competencias de extensión universitaria y de gestión académica. En contraste, los docentes priorizan las competencias de gestión académica y las investigativas. Para los estudiantes, la competencia de menor importancia en el proceso de evaluación del desempeño docente lo constituye la competencia investigativa. Esto revela coincidencia con los resultados obtenidos en otras investigaciones precedentes realizadas por Cortés et al. (2014) donde se afirma que al estudiante principalmente le interesa lo que logra el docente con ellos y secundariamente otros aspectos.

\section{CONCLUSIONES}

El análisis de las percepciones de estudiantes y docentes sobre el instrumento de evaluación del desempeño docente en una universidad pública en el contexto angolano arrojó que las dimensiones que deben emplearse para esta evaluación son: las competencias pedagógico-didácticas, investigativas, de extensión universitaria y de gestión académica.

De acuerdo con las percepciones de estudiantes y docentes, la competencia que priorizan en la evaluación del desempeño docente es la competencia pedagógico-didáctica, en otras palabras que el profesor facilite el aprendizaje. Sin embargo, no hay coincidencia de criterios en cuanto al orden de importancia que le confieren a las demás dimensiones. Los estudiantes consideran que deben valorarse las competencias de extensión universitaria, de gestión académica e investigativas en este orden de prioridad; mientras los docentes priorizan las competencias de gestión académica, las investigativas y de extensión universitaria.

\section{REFERENCIAS}

Cancino, V. E. y Márquez, T. S., Evaluación de Desempeño de la Función Académica: Análisis de un Sistema en el Contexto Universitario Chileno, doi: 10.4067/S0718-50062015000300005, Formación Universitaria, (en línea), 8(3), 35-46 (2015)

Cardoso, E., Cerecedo, M. y Vanegas, E. (2013). Las competencias docentes en los programas de posgrado en administración: Un estudio diagnóstico, doi: 10.4067/S0718-50062013000200006, Formación Universitaria, (en línea), 6(2), 43 - 50 (2013)

Cochram, W. G., Técnicas de muestreo. Compañía Editorial Continental, S.A. México (1999)

Coelho, J. G., y Vásquez-Rizo, F. E., Del otro lado de la pizarra: relación estudiante-profesor desde perspectivas disciplinares, Rev. Educación y Educadores, ISSN: 0123-1294, (en línea), 11(1), 103-126, 2008. http://www.scielo.org.co/pdf/eded/v11n1/v11n1a07.pdf. Acceso: 12 de noviembre (2015)

Cortés, E., Campos, M., y Moreno, P. M., Priorización de las dimensiones de evaluación del desempeño docente por el estudiante, en tres áreas del conocimiento, doi: 10.4067/S0718-50062014000200002, Formación universitaria, (en línea),7(2), 3-10 (2014)

Creus, A., Padilla P. P., y Sancho, J. M., Docencia, investigación y gestión en la universidad: Una profesión tres mundos, Rev. Praxis Educativa, ISSN: 0328-9702 (en línea), 16(14), 17-34, 2011. http://www.biblioteca.unlpam.edu.ar/pubpdf/praxis/n14a03sancho.pdf. Acceso: 06 de Diciembre (2015)

Cruz, M. A. V., Arroyo, G. C., Barajas, Y. E. L., Análisis comparativo de criterios de desempeño professional para la enseñanza en cuatro paises de America, Rev. Actualidades Investigativas en Educación, ISSN: 14094703, (en línea), 14(3), 4, 2014. http://www.revista.inie.ucr.oc.cr/. Acceso: 25 de noviembre (2015)

Chimpolo, J. M. F., Tecnología para la gestión de recursos humanos en instituciones de educación superior angolanas. Caso de la Universidad Agostinho Neto. Tesis presentada en opción al grado científico de doctor en ciencias técnicas en la Universidad Holguín, Cuba (2015) 
Daura, F., Las estrategias docentes al servicio del desarrollo del aprendizaje autorregulado, Rev. Estudios Pedagógicos, ISSN: 0718-0705, (en línea), 37(2), 77-88, 2011.

http://www.scielo.cl/pdf/ested/v37n2/art04.pdf. Acceso: 06 de diciembre (2015)

De León, A. T., y Huerta, J. M., Cuadro de mando integral para el diseño y validación de instrumentos para valorar el desempeño académico de docentes, Avaliação, Campinas; Sorocaba, SP: 15(1), 57-72 (2010)

De Veaux, R. D., Elements of Experimental Design. International Thompsom Editores, S.A. México (2001)

Fernandes, D.J., Martínez, C.C.M., Sotolongo, M., Desempenho docente. Contribuição para o processo de integração dos estudantes que ingressam no Ensino Superior. Caso do Instituto Superior de Ciências de Educação do Huambo, República de Angola, Rev. Órbita Pedagógica, ISSN: 2409-0113 (en línea), 2(1), 112, 2015. http://revista.isced-hbo.ed.ao/index.php/rop. Acceso: 10 de diciembre (2015)

Fernández, A., La innovación de la docencia como estrategia de cambio ante los desafíos del EEES. Universidad Politécnica de Valencia. Experiencias Docentes y Tics. ICE. Universidad de Oviedo. $1^{1 \underline{a}}$ edición, 7-341. Ediciones Octaedro, Barcelona, España (2011)

Galvão, H.M., Corrêa, H.L., Alves, J.L., Modelo de avaliação de desempenho global para instituições de ensino superior. Universidade Federal de Santa-Clara, Brasil, Rev. de Administração da Universidade Federal de Santa Maria, ISSN: http://www.spell.org.br/documentos/ver/7451/modelo-de-avaliacao-dedesempenho-global-para-instituicao-de-ensino-superior/i/pt-br (en línea), 4(1), 425-441, 2011. http:// www.redalyc.org/articulo.oa?id=273421614008. Acceso: 12 de noviembre (2015)

Gomes, J. F. S. y Cesário, F., Investigação em gestão de recursos humanos, ISBN: 978-972-592-420-4, 181-198, Escolar Editora, Lisboa, Portugal (2014)

Marsh, H. W., Students' evaluations of university teaching: dimensionality, reliability, validity, potential biases and usefulness (en línea), 319-383, 2007. http://www.eva.um.ac.ir/43/maghale/18.pdf. Acceso: 28 de Abril (2015)

Martínez, J. F., Combing multiple measures of teacher practice and performance: technical and conceptual considerations for teacher evaluation, Rev. de Investigación Educación Latinoamericana, ISSN: 0719-0409 (en línea), 50(1), 4-20, 2013. http://www.pensamientoeducativo.uc.cl/files/journals/2/articles/528/public/5281467-1-PB.pdf. Acceso: 12 de noviembre (2015)

Martínez, M.A., Cegarra, N.J.G., Rubio, S.J.A., Aprendizaje basado en competencias: una propuesta para la autoevaluación docente, Rev. de Curriculum y Formación del Profesorado, ISSN: 1989-639 (en línea), 16(2),373-386,2012. http://www.ugr.es/local/recfpro/rev162COL5.pdf. Acceso: 16 de diciembre (2015)

Mas, T. O., Las competencias investigadoras del profesorado universitario: la percepción del propio protagonista, de los alumnos y de los expertos, Rev. de Curriculum y Formación del Profesorado, ISSN: 1989-639, (en línea), 18(3),256-273, 2014. http://www.ugr.es/local/recfpro/rev183COL4.pdf. Acceso: 21 de noviembre (2015)

Matos, T. A., Quispe, Y. W., Evaluación de las competencias docentes en maestros de matemática de PUNO, Rev. de Curriculum y Formación del Profesorado, ISSN: 1989-639, (en línea), 18(1), 257-278, 2012. http://www.ugr.es/local/recfpro/rev162COL4.pdf. Acceso: 6 de octubre (2015)

Morán, R., Cardoso, E. O., Cerecedo, M. T. y Ortíz, J. C., Evaluación de las Competencias Docentes de Profesores Formados en Instituciones de Educación Superior: El Caso de la Asignatura de Tecnología en la Enseñanza Secundaria, doi: 10.4067/S0718-50062015000300007, Formación Universitaria, (en línea) 8(3), 57-64 (2015)

Moreno, M. P.et al., Los Comentarios Abiertos como Referente de Evaluación de la Docencia Universitaria: La Conveniencia de su Interpretación y Tratamiento, doi: 10.4067/S0718-50062014000100006, Formación Universitaria, (en línea) 7(1), 41-48 (2014)

Murillo, F.J. Y Hernández, R., Para uma avaliação do desempenho docente não universitário em espanha. Lições aprendidas de directores/as escolares, Rev. Iberoamericana de Evaluación Educativa, ISSN: 1989-0397, (en línea), 5(1), 367, 2012. http://www.rinace.net/riee/numeros/vol5-num1_e/art28.pdf. Acceso: 25 de octubre (2015)

Nascimento, A., Linhas Mestras para a Melhoria da Gestão do Subsistema do Ensino Superior.Luanda, Angola: Diario da Republica (2005) 
Nava, M. y Rueda, M., La evaluación docente en la agenda pública, Rev. Electrónica de Investigación Educativa, (en línea), 16(1), 1-11, 2013. http://www.redie.uabc.mx/vol16no1/contenido-nava-rueda.html. Acceso: 16 de noviembre (2015)

Ospina, A., Currículo por competencias en la Universidad de La Sabana, Rev. Aquichan, ISSN: 1357-5997 (en línea), 6(1), 117-124, 2006. http://www.scielo.org.co/pdf/aqui/v6n1/v6n1a11.pdf. Acceso: 6 de Octubre (2015)

Páramo, P., Factores psicosociales asociados a la evaluación del docente, Rev. Educación y Educadores, Rev. Educación y Educadores, ISSN: 0123-1294, (en línea), 11(1) 11-30, 2008. http://educacionyeducadores.unisabana.edu.co/index.php/eye/article/viweArticle/715. Acceso: 25 de octubre (2015)

Pimienta, J. H., Elaboración y validación de un instrumento para la medición del desempeño docente basado en competencias, REDU: Rev. de Docencia Universitaria, número monográfico dedicado a Equidad y Calidad en la Docencia Universitaria: Perspectivas Internacionales, ISSN: 1887-4592 (en línea), 2, 231250, 2014. http://www.red-u.net/. Acceso: 18 de noviembre (2015)

Portugal, M. N., Machado, D. M. R., y Machado, A. R., Organizações. Introdução à gestão e desenvolvimento das pessoas, ISBN: 978-972-592-414-3, 1를 edición, 133-143, Escolar Editora, Lisboa, Portugal (2014)

Rocha, C.R., Escala de opinión de los estudiantes sobre la efectividad de la docencia (EOEED) en Educación Superior, doi: 10.4067/S0718-50062013000600003, Formación Universitaria, (en línea), 6(6), 13$22(2013)$

Sandoval, Y. V., La evaluación de las funciones docentes en los proyectos de acción social de la universidad de Costa Rica: Análisis y propuesta de actualización, Rev. Actualidades Investigativas en Educación, ISSN: 1409-4703, (en línea), 14(3), 2, 2014. http://www.revista.inie.ucr.oc.cr. Acceso: 12 de noviembre (2015)

Shiroma, E. O. y Schneider, M. C., Avaliação de desempenho docente: contradições da política "para poucos" na era do "para todos", doi: http://dx.doi.org/10.5216/ia.v38i1.25131, Rev. Faculdade de Educação da UFG, (en línea), 38 (1), 89-107, (2013)

Tirado Segura, F., Miranda Díaz, A., y Sánchez Miguel, A., La evaluación como proceso de legitimidad: la opinión de los alumnos. Reporte de una experiencia, Rev. Perfiles educativos, ISSN: 0185-2698, (en línea), 29(118), 7-24, 2007. http://www.redalyc.org/articulo.ao?id=13211802. Acceso: 20 de diciembre (2015)

Tobón, S. et al., Secuencias didácticas: aprendizaje y evaluación de competencias, ISBN: 978-607-442-909$1,1^{a}$ edición, Pearson Educacional, México (2010)

Tobón, S., Competencias en la Educación Superior. Políticas hacia la calidad, ISBN: 978-958-648-826-6, ECOE Ediciones, Bogotá, Colombia (2006)

Tobón, S., Formación integral y competencias, ISBN: 978-958-648-826-6, 4aㅗ edición, 227, ECOE ediciones, Bogotá, Colombia (2013)

Torra, I. et al., Identificación de competencias docentes que orienten el desarrollo de planes de formación dirigidos a profesorado universitario. Rev. Docencia Universitaria, ISSN: 1887-4592 (en línea), 10(2), 21-56, 2012. http://www.red-u.net/redu/documentos/vol10_n2_completo.pdf. Acceso: 25 de abril (2016)

Zabala, A.; Arnua, L., La enseñanza de las competencias. Rev. Aula de innovación educativa, ISSN: 20144725 (en línea), 161, 40-46, 2007.

http://upvv.clavijero.edu.mx/cursos/ObservacionPracticall//vector2/tarea6/documentos/Zabala_Arnua.pdf.

Acceso: 03 de mayo (2016)

Zabalza, M., LA universidad de las competencias. Rev. Docencia Universitaria, ISSN: 1887-4592 (en línea), 10(2), 21-56, 2012. http://www.red-u.net/redu/documentos/vol10_n2_completo.pdf. Acceso: 25 de abril (2016) 